\title{
Using Magnetic Resonance Perfusion to Stratify Overall Survival in Treated High-Grade Gliomas
}

\author{
Crystal Fong, Sameer Parpia, Brian Yemen, Scott Tsai, Jeffrey Greenspoon
}

\begin{abstract}
Background: MR perfusion imaging is a relatively new technique that may aid in identifying recurrent tumor (RT) in those with radically treated high-grade gliomas (HGG). We aim to assess the relationship between dynamic susceptibility contrast-enhanced MR perfusion (DSC-MRP) and overall survival to establish a baseline for future research and to determine the utility of DSC-MRP as a clinical decision-making and prognostic tool. Methods: We conducted a retrospective cohort study. Adults with pathologically confirmed HGG at the Juravinski Cancer Centre, Ontario between January 2011 and April 2014 with at least one post-treatment DSC-MRP were included. DSC-MRP was interpreted as positive or negative for tumor recurrence by experienced radiologists. The primary outcome was overall survival. Results: Sixty-one patients were enrolled. Median survival for patients with a positive DSC-MRP scan was 4.5 months compared with 10.2 months for those with a negative DSC-MRP scan (hazard ratio [unadjusted] $=2.51 ; 95 \%$ confidence interval $=1.10-5.67 ; p$-value $=0.03$ ). Multivariable modeling (adjusted) that included all pre-selected variables showed similar results. Conclusion: Survival time in patients with HGG is generally low, and almost all patients will demonstrate RT. Our data suggest a positive DSC-MRP correlates with lower overall survival and may signify the presence of highly active RT. These results generate a hypothesis that there may be a prognostic role for the use of serial DSC-MRP for tumor surveillance. More importantly, this biomarker may aid in decision making for treatment plans and palliation.
\end{abstract}

RÉSUMÉ: Utiliser l'IRM de perfusion pour déterminer la survie globale de patients traités pour des gliomes de haut grade. Contexte : L'IRM de perfusion est une technique relativement nouvelle qui pourrait aider à identifier la récurrence tumorale dans le cas de gliomes de haut grade. Notre objectif est d'évaluer l'association pouvant exister entre les IRM de perfusion dynamique de contraste de susceptibilité (dynamic susceptibility contrast-enhanced MR perfusion) et la survie globale des patients afin d'établir des lignes directrices pour la recherche à venir et de déterminer l'utilité diagnostique de cette technique d'imagerie en ce qui concerne la prise de décisions cliniques. Méthodes : Nous avons ainsi effectué une étude de cohorte rétrospective incluant des patients adultes atteints de gliomes de haut grade. Ces patients du Juravinski Cancer Centre (Ontario) ont vu leur maladie être confirmée entre janvier 2011 et avril 2014. À la suite d'un traitement dans cet établissement, ils ont aussi bénéficié d'au moins une IRM de perfusion dynamique de contraste de susceptibilité. Un tel examen médical a été jugé positif ou non par des médecins radiologues expérimentés en tenant compte de la réapparition de tumeurs. Enfin, le principal indicateur évalué a été la survie globale des patients. Résultats : Soixante et un patients ont fait partie de cette étude. La survie globale médiane des patients dont l'IRM de perfusion dynamique de contraste de susceptibilité était positive était de 4,5 mois comparativement à 10,2 mois pour ceux dont la même IRM était négative (rapport de risque [sans ajustement] =2,51; IC 95\%=1,10 à 5, $67 ; p=0,03$ ). Une approche de modélisation multi-variable (avec ajustement) ayant inclus toutes nos variables présélectionnées a produit des résultats semblables. Conclusion : La survie globale des patients atteints de gliomes de haut grade est généralement faible ; de plus, presque tous les patients vont finir par donner à voir une récurrence tumorale. Nos données suggèrent donc que les patients dont l'IRM de perfusion dynamique de contraste de susceptibilité était positive peuvent être associés à une survie globale plus faible, ce qui pourrait signifier la présence d'une tumeur récurrente fortement active. Ces résultats laissent à penser que l'IRM de perfusion dynamique de contraste de susceptibilité pourrait jouer un rôle diagnostique dans le suivi des tumeurs. Plus important encore, il est possible que ce biomarqueur soit utile dans le processus décisionnel qui concerne les plans de traitement et les soins palliatifs.

Keywords: MR perfusion, Glioma, Tumor imaging, Cerebral blood volume

doi: $10.1017 / \operatorname{cjn} .2019 .225$

Can J Neurol Sci. 2019; 46: 533-539

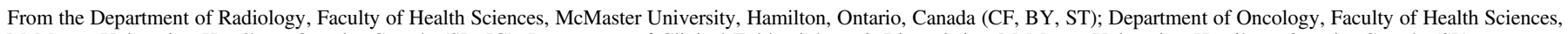
McMaster University, Hamilton, Ontario, Canada (SP, JG); Department of Clinical Epidemiology \& Biostatistics, McMaster University, Hamilton, Ontario, Canada (SP)

Received July 28, 2016. Final Revisions Submitted April 24, 2019. Date of Acceptance May 27, 2019.

Correspondence to: Crystal Fong, Department of Radiology, McMaster University, 1280 Main St. W, L8S 4L8, Hamilton, ON, Canada. Email: crystal.fong@medportal.ca 


\section{INTRODUCTION}

The prevalence of malignant gliomas worldwide is uncommon with a quoted annual incidence of 5/1,00,000 (,2 $^{1,2}$ However, it accounts for disproportionately high morbidity and mortality. ${ }^{1}$ Outcomes are poor with the most important adverse prognostic factors being advanced age, aggressive histologic features, poor Karnofsky Performance Status (KPS), and unresectable tumors. 1,2

For a primary diagnosis of high-grade gliomas (HGG), the standard treatment is maximum safe surgical resection of tumor with radiotherapy and temozolomide. ${ }^{3}$ However, despite optimal treatment, almost all malignant gliomas recur, with a median time to progression for the most common entity, glioblastoma, averaging 6.9 months. ${ }^{1}$ The treatment for recurrence is controversial but may involve repeat resection, focal irradiation, and systemic therapy. Unfortunately, the efficacy of these treatments remains questionable. ${ }^{4,5}$ Since treatments are not entirely benign, there should be consideration for ongoing supportive care instead if disease progression is not confirmed. Tumor surveillance becomes, then, very important to determine whether there is true recurrence and how a managing team should proceed. ${ }^{5}$

Surveillance thus far has been via serial conventional MRI with gadolinium enhancement. The Response Assessment in Neuro-Oncology (RANO) criteria, published in 2010 as an update to the MacDonald criteria, is used to assess disease progression and treatment response in glioblastoma. ${ }^{6}$ However, despite the strict criteria, findings often remain indeterminate, especially within the first year after treatment. ${ }^{7}$ There is considerable overlap in the findings seen on conventional MRI between recurrent tumor (RT) and those seen with treatmentrelated necrosis $(\mathrm{TN})$, both usually demonstrating new or increased enhancement. ${ }^{8}$ Within 1-month post radiotherapy, up to $40 \%$ of conventional MRIs demonstrate increased enhancement, and in $50 \%$ of those cases, this is related to transient increase in vessel permeability which improves over time, a phenomenon called "pseudoprogression". 9 Some have gone as far as to say that the presence of TN is based on the timing of MRI rather than the findings, with TN classically seen from 2 to 32 months post treatment (most within 2 years) while a new abnormality occurring after 3 years is very unlikely to be related to necrosis. ${ }^{7}$

Recent literature has suggested that if an accurate test to distinguish RT from TN within the first couple of years after primary treatment were available, there may be utility in determining prognosis and targeting salvage therapies to change quality of life. ${ }^{10-12}$ Increasingly, certain advanced MRI techniques, including diffusion-weighted imaging, diffusion tensor imaging, perfusion imaging, and proton magnetic resonance spectroscopy are being looked at as means of monitoring response to therapy. ${ }^{13-18}$ At our institution, perfusion imaging via dynamic susceptibility contrast-enhanced MR perfusion (DSC-MRP) is used, with the assumption that RT should demonstrate increased perfusion due to neovascularity compared with $\mathrm{TN}$, which would demonstrate similar or decreased perfusion when compared to normal contralateral brain parenchyma. $^{17}$

Several authors have looked at perfusion imaging as a technique for identifying histopathological changes in treated HGG, and multiple small studies have shown it to be a valuable biomarker for RT, differentiating it from mimickers such as TN. ${ }^{10,14,17,19-23}$

Others have demonstrated that perfusion imaging can also be a tool to establish tumor grade or monitor change in tumor grade ${ }^{24-26}$ some have even explored perfusion imaging at a molecular and genomic level to establish tumor subclassification. ${ }^{27}$ However, the correlation between DSC-MRP and clinical outcomes such as overall survival remains to be evaluated. Geer et al. ${ }^{11}$ looked at the addition of DSC-MRP to conventional MRI when following patients with glial tumors and demonstrated increased confidence for both neuroradiologists and neuro-oncology teams in the diagnosis of recurrence; however, a direct comparison with patient outcomes was not conducted. SanzRequena et al. ${ }^{12}$ looked at survival times in those with HGG; however, they focused on the relationship between different quantitative parameters calculated from DSC-MRP data and overall survival rather than on the interpretation of the entire perfusion study as a whole, which would be more representative of current clinical practice. More recently, Mangla et al. ${ }^{10}$ looked at changes in the well-established perfusion parameter, relative cerebral blood volume (rCBV), and its relationship to overall survival, but in this study, all patients received DSC-MRP 1-month post treatment rather than at the time of suspected recurrence, which again is less clinically applicable, and may not be reflective of the true utility of DSC-MRP in clinical decision-making.

We hypothesize that among patients with treated HGG (WHO grade III and IV) and indeterminate clinical and conventional MRI findings for tumor recurrence, DSC-MRP may act as a clinically useful test to stratify overall survival, with a positive result correlating to decreased overall survival when compared to a negative result.

\section{Materials And Methods}

A retrospective search of the Picture Archiving and Communication Systems data was conducted at the Juravinski Cancer Centre (Hamilton, ON) between January 2011, when MR perfusion imaging was initiated and April 2014 to include all patients who had received DSC-MRP. At our institution, this group that received DSC-MRP encompassed a population with suspected RT based on clinical exam that is well enough to be considered for salvage therapy. Patients were eligible to be included in the study if they were greater than 18 years of age and had a histopathologically confirmed new diagnosis of HGG (WHO grade III or IV), including glioblastomas, anaplastic oligodendrogliomas, grade III astrocytomas, and other mixed HGG. All patients were treated with maximum surgical resection or biopsy, usually followed by the standard treatment of radiation therapy with or without concurrent and adjuvant chemotherapy. All patients received a pre-operative CT head or MRI head to evaluate the extent of disease and a post-treatment gadoliniumenhanced conventional MRI.

Patients were excluded if they had treatment with an antiangiogenesis agent, such as bevacizumab at the time of DSCMRP. Anti-angiogenic agents can lead to findings of pseudoresponse by decreasing vascular permeability and normalizing an otherwise abnormal blood-brain barrier, falsely altering perfusion dynamics. $^{28}$ 
Table 1: Baseline characteristics of the study population

\begin{tabular}{|c|c|}
\hline Characteristic & All subjects $(n=61)$ \\
\hline Age (years): mean (SD) & $56(11)$ \\
\hline KPS: mean (SD) & $76(16)$ \\
\hline Tumor size $(\mathrm{cm})$ : mean $(\mathrm{SD})$ & $4.4(1.6)$ \\
\hline \multicolumn{2}{|l|}{ Gender: $n(\%)$} \\
\hline Male & $35(57)$ \\
\hline Female & $26(43)$ \\
\hline \multicolumn{2}{|l|}{ Tumor grade: $n(\%)$} \\
\hline III & $18(30)$ \\
\hline IV & $43(70)$ \\
\hline \multicolumn{2}{|l|}{ Extent of resection: $n(\%)$} \\
\hline Total & $32(53)$ \\
\hline Subtotal & $21(34)$ \\
\hline Biopsy & $8(13)$ \\
\hline \multicolumn{2}{|l|}{ Treatment: $n(\%)$} \\
\hline Surgery, radiation, chemotherapy & $52(85)$ \\
\hline Radiation, chemotherapy only & $1(2)$ \\
\hline Radiation only & $8(13)$ \\
\hline \multicolumn{2}{|l|}{ RANO: $n(\%)$} \\
\hline Complete response & $2(3)$ \\
\hline Partial response & $13(22)$ \\
\hline Stable disease & $16(27)$ \\
\hline Progression & $29(48)$ \\
\hline Data not available* & $1(2)$ \\
\hline $\begin{array}{l}\text { Time from presentation to perfusion study } \\
\text { (months): mean (SD) }\end{array}$ & $12(10)$ \\
\hline
\end{tabular}

*One subject was missing the initial post-operative imaging to determine RANO status.

Clinical variables with the strongest known association with overall survival such as patient age and gender, KPS at presentation, extent of surgical resection, and initial tumor grade were obtained. ${ }^{1,9}$ If KPS was provided as a range of numbers in the clinical notes, the lowest score was taken. Extent of resection was based on surgical notes rather than on post-operative imaging, as there was substantial variability in the timing and interpretation for post-operative imaging. Some patients were brought to us from an outside institution, and MRI sequences were hetergeneous. Additional information obtained included date of presentation, date of initial radiation treatment, and date of DSC-MRP. If the exact date of presentation was not specified in the clinical notes, then the first of the month of presentation was chosen. The baseline characteristics of the study population are included in Table 1.

Finally, survival data was collected from various sources, including medical records and obituary databases. Those whose dates of death were unable to be obtained (15 in total) were presumed to have passed away 3 months post initiation of palliative care. This timeframe was based on the difference between the median overall survival and median progression- free survival times in the literature and extrapolated from the time spent in hospital after progression-free survival.

Authorized consent from the Hamilton Integrated Research Ethics Board was obtained for the inclusion of patients in this study.

\section{MRI Acquisition and Interpretation}

All MRI studies were acquired on a 3.0T MR system (GE Discovery MR750) using a standard 32 channel head coil and assessed by one of the two radiologists with experience in interpreting DSC-MRP data. MR imaging protocol included axial and sagittal T1 FLAIR, axial and coronal FSE T2, axial T2 FLAIR, DWI, and contrast-enhanced axial FSPGR T1 and T1 FLAIR images. A T2*-weighted DSC perfusion sequence was performed with the following parameters at our institution: axial plane, TR $2000 \mathrm{~ms}$, TE $20 \mathrm{~ms}$, acquisition matrix $96 \times 128,28$ slices with slice thickness $5 \mathrm{~mm}$, FOV $22 \mathrm{~cm}, 34$ dynamics. The contrast agent used was gadobutrol $\left(\right.$ Gadovist $\left.^{\circledR}\right)$, dosed at 0.1 $\mathrm{mmol} / \mathrm{kg}$ and power injected after 6-second delay, at a rate of 2.5 $\mathrm{mL} / \mathrm{s}$ followed by a $30-\mathrm{mL}$ saline bolus. Post processing to generate color maps based on cerebral blood volume (CBV) was performed by the MR technologist on a GE AW workstation using the GE Brainstat software. CBV was calculated by integrating the negative enhancement portion of the $\mathrm{T} 2 *$-weighted Signal Intensity-Time Curve (T2SITC).

A region of interest (ROI) was selected by the radiologist on the conventional MRI post-gadolinium enhanced T1 images (Figure 1A and B). The ROI was then compared to the normal contralateral brain parenchyma at the same level to determine the relative cerebral blood volume (rCBV). Increased $\mathrm{rCBV}$ equated to a positive DSC-MRP while equal or decreased $\mathrm{rCBV}$ equated to a negative DSC-MRP.

\section{Statistical Analysis}

The overall survival of those with positive (increased rCBV) and negative (similar or decreased rCBV) DSC-MRP scans was estimated using the Kaplan-Meier method and compared using the log-rank test. Unadjusted hazard ratio was estimated using the Cox model with the DSC-MRP scan result alone. Adjusted hazard ratio was estimated using a similar approach but included the DSC-MRP scan result as well as patient age, gender, KPS, histologic grade, and extent of resection.

Unfortunately, the timing of DSC-MRP was highly variable, as there is no standard time for the ordering of this test, and it is largely based on clinical suspicion for RT. To minimize guaranteed-time bias, the observation start time was chosen as the date of the DSC-MRP scan, presuming that patients who were asymptomatic did not have significant tumor recurrence prior to the first DSC-MRP. In addition, we performed a sensitivity analysis with presentation date as the start of the observation period and DSC-MRP scan as a time-dependent variable in the Cox model, to ensure that this time-adjusted analysis yielded similar results.

\section{Results}

A total of 61 patients with treated HGG who received at least one post-treatment DSC-MRP were eligible and included in the 
(A)

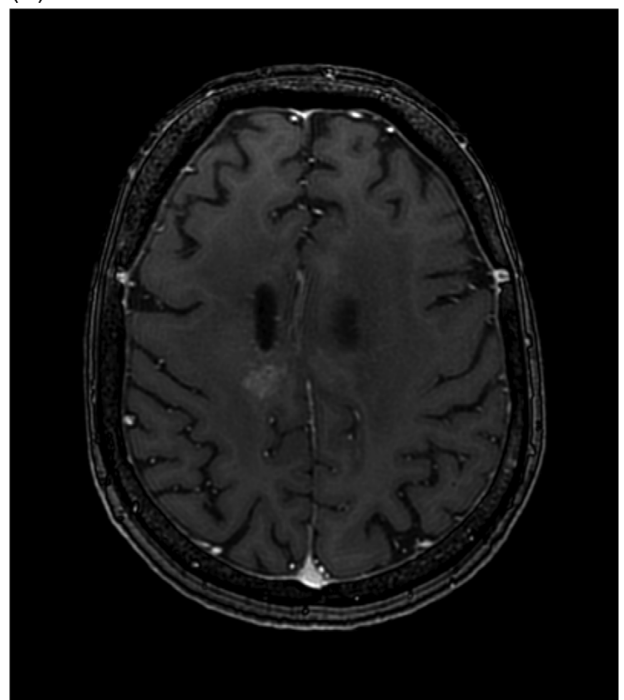

(B)

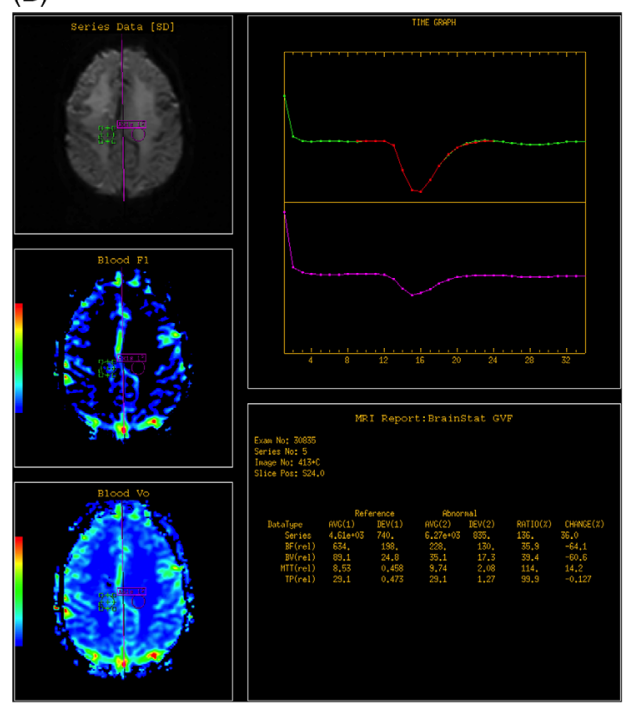

Figure 1. (A) Axial post-gadolinium enhanced $T 1$ fat saturated image of the brain demonstrates abnormal heterogeneous enhancement within the high right frontoparietal lobe, adjacent to the site of previous resection. (B) DSC-MRP was determined by assessing a region of interest based on the site of abnormal enhancement, as determined by an experienced radiologist (green circle) and compared to the contralateral normal brain parenchyma (purple circle). Graph depicts the change in perfusion over time for the two ROIs and the calculated values are used to generate color maps.

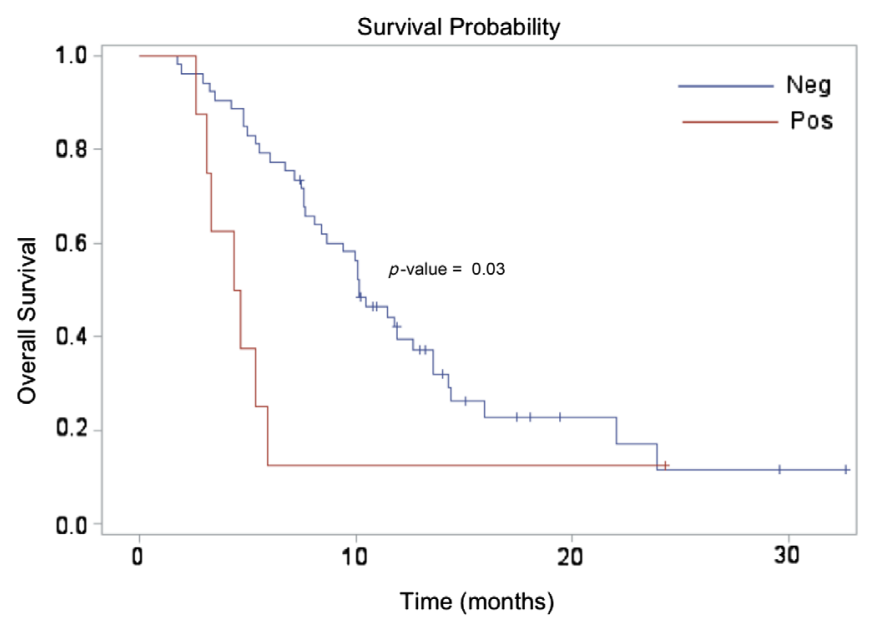

Figure 2. Kaplan Meier survival estimate demonstrating a significant difference between the perfusion-negative (median survival 10.2 months) and perfusion-positive (median survival 4.5 months) cohorts.

analysis. Four patients were excluded due to the use of bevacizumab at the time of scan. Fifty-three $(87 \%)$ patients had a negative DSC-MRP and eight $(13 \%)$ had a positive DSC-MRP.

The median survival for the entire cohort was approximately 10 months, which is slightly higher than what has been reported in the literature for those with suspected recurrent high-grade glioma (3-6 months). ${ }^{28}$ This unexpected finding may be related to our case selection, as we only included patients who received DSC-MRP - which, at our institution, are only those well enough to be considered for salvage therapy. Median survival for patients with a positive DSC-MRP scan (4.5 months) was significantly lower compared to those with a negative DSC-MRP scan (10.2 months), [hazard ratio $(\mathrm{HR})=2.51 ; 95 \%$ confidence interval
$(\mathrm{CI})=1.10-5.67 ; p=0.03]$. (Figure 2) The adjusted analysis including all pre-selected variables (age, gender, KPS, tumor grade, extent of surgical resection) demonstrated a similarly significant relationship $(\mathrm{HR}=2.53,95 \% \mathrm{CI}: 1.05-6.14 ; p=0.04)$.

The mean and standard deviation (SD) for duration of time between presentation and DSC-MRP scan date was 13 months $(\mathrm{SD}=11)$ for the DSC-MRP negative patients and 10 months $(\mathrm{SD}=4)$ for the DSC-MRP positive patients. However, sensitivity analysis adjusting for this showed similar results to the non-timedependent analysis.

\section{Discussion}

Median survival in those with recurrent HGG is low, and quality of life is quick to diminish. ${ }^{1}$ Determining the presence of RT is imperative to ensure both longevity and maintained quality of life. Despite recent advances in neuro-imaging, an accurate method of tumor surveillance remains elusive. ${ }^{17}$

To our knowledge, our study is the first to directly evaluate the correlation between DSC-MRP and overall survival in patients with treated HGG and suspected recurrence but indeterminate clinical and conventional MRI findings using real-life clinically applicable data. Our results demonstrate a statistically significant relationship between DSC-MRP and overall survival: patients with positive DSC-MRP have lower survival times and a 2.5 times increased risk of death compared to those with negative DSC-MRP. This result is consistent with the expected angiogenesis and neovascularity seen in true RT.

Our findings suggest two roles for using DSC-MRP to monitor those with HGG. First, it may act as an imaging biomarker for high tumor aggressiveness, regardless of histopathology, which can be limited by sampling; second, its relationship with overall survival can serve as a prognostic and thus, clinical decision-making, tool. ${ }^{34}$ 
How perfusion imaging can act as an appropriate biomarker for malignancy is based on the physiologic changes seen with tumor angiogenesis. DSC-MRP relies on the administration of a paramagnetic contrast agent during the acquisition of magnetic resonance images. During the first pass of the contrast agent, the distribution is mainly intravascular, resulting in maximal concentration gradient between the intra- and extravascular compartments, reflecting tissue perfusion with minimal diffusivity. The paramagnetic agent causes a transient change in tissue magnetic field and elicits a signal change in the acquired images. Thus, perfusion imaging is particularly useful in detection of highly malignant tumors with abnormally increased vascular networks. In contrast, the CBV remains the same or decreased in $\mathrm{TN}$, as it is dependent on the distribution of blood capillaries rather than vascular permeability. ${ }^{23}$ These findings differ from conventional MRI, where both tumor angiogenesis and damage from radiation injury manifest with new enhancement. ${ }^{28}$

Unfortunately, the limitation to perfusion imaging is that interpretation is largely variable and often inconclusive. Although CBV itself can be quantitatively measured, the selection of an ROI to calculate the ratio for $\mathrm{rCBV}$ is based on findings of new or increased enhancement adjacent to the surgical cavity on the conventional MRI - and is often radiologist-dependent. Additionally, the final interpretation of the DSC-MRP is based on generated color maps from computed rCBV values, which is non-quantitative and leaves room for subjective opinions.

Quantitative upper and lower limit rCBV values have been proposed to differentiate between RT and TN. An early study of perfusion MRI showed that an $\mathrm{rCBV}$ ratio of greater than 2.6 likely represented RT and a value of less than 0.6 favored radiation necrosis. ${ }^{22}$ However, these cutoff levels leave a broad range of values in an "intermediate" zone, resulting in inconclusive data. And in fact, assessment of perfusion is often limited due to overlapping findings of mixed viable tumor and necrosis on pathological exam. Additionally, hyperplastic dilated vasculature in necrosis can occur, and disruptions of the blood-brain barrier can interfere with accurate measurements of perfusion. ${ }^{7} \mathrm{Hu}$ et al. ${ }^{29}$ prospectively evaluated 42 specimens using threshold rCBV values and suggested a rCBV cutoff value of 0.71 to optimize the difference between RT and posttreatment effect. Gasparetto et al. ${ }^{30}$ retrospectively evaluated 30 patients with HGG and found an rCBV cutoff value of 1.8 to be the most useful distinguishing point. These quantitative values are currently not used in clinical practice, and the use of rCBV color maps is a more practical application.

Our study confirms the utility of even a subjective interpretation of DSC-MRP, demonstrating a correlation with outcomes as expected. Secondarily, the study results serve as an internal audit of the quality of DSC-MRP interpretation at our institution. Future studies could benefit by looking at the quantitative rCBV values in comparison with subjective interpretation of color maps and within the context of overall survival as a clinical endpoint. Additional histopathologic correlation would also help to validate the DSC-MRP technique used at our institution but unfortunately it was not always available.

Other quantitative perfusion parameters that have been shown to be effective markers of RT in early studies and can be further evaluated include relative peak height $(\mathrm{rPH})$ and relative percent of signal recovery (rPSR). ${ }^{8,14,31,32}$ The $\mathrm{rPH}$ is determined by subtracting the minimum signal intensity on the T2SITC from the peak height of the pre-contrast signal intensity, which is then normalized to the peak height of the normal contralateral white matter. ${ }^{31}$ As expected, $\mathrm{rPH}$ has been shown to be usually higher in RT compared to TN, with one group showing a cutoff value of 1.38 to have $89 \%$ sensitivity and $81 \%$ specificity for tumor recurrence. ${ }^{8}$ Finally, the rPSR, essentially the ratio between the difference of signal intensity post- and pre-contrast and the difference in normal brain signal intensity post- and pre-contrast, has been less well studied. ${ }^{31}$ Barajas et al. ${ }^{14}$ used a cutoff of $87.3 \%$, with increased rPSR more likely to denote TN, and achieved a sensitivity of $78.26 \%$ and specificity of $76.19 \%$ for differentiating RT from TN. More recently, some have looked at cerebral blood flow (CBF) in addition to CBV to augment the distinction between RT and $\mathrm{TN}^{21,26,33}$ The role of the other MRP parameters and their relationships with overall survival is yet to be determined.

In addition to being a tumor biomarker, our results suggest a secondary role for perfusion imaging to function as a prognostic and clinical decision-making tool. To extrapolate, there may even be a role for serial DSC-MRP in place of conventional MRI. Patients may follow a predicted survival curve with serially negative DSC-MRP, but convert to the more aggressive perfusion-positive survival curve with true recurrence, defining a time point for histopathologic conversion to highly active tumor and for imminent clinical change. These serial tests would help managing neuro-oncology teams to optimize treatment and palliation as salvage therapies evolve and become more effective.

Regrettably, at this time, most diagnostic imaging departments do not have the capacity to perform serial perfusion MR imaging on all patients for tumor surveillance, but the hope is that with the accumulation of evidence for the utility of DSC-MRP, this practice will become a reality, and the future directed research can compare single DSC-MRP to serial DSC-MRP imaging in a prospective manner to fully elucidate its clinical value.

There are a number of limitations in our study, including its retrospective nature and small sample size, attributable to the rarity of these tumors and the relative novelty of DSC-MRP. The small sample size, in particular of the perfusion-positive group $(n=8)$, decreases the power of our study and makes the results less robust and subject to confounding variables. We chose to include five pre-determined variables in our analysis, including age and KPS, the two most strongly known independent predictors of overall survival; however, we must keep in mind that there are several other variables, including tumor size, RANO status, primary treatment, and use of salvage therapy which were not included due to the small sample size and whose effect on survival rates is unknown. Additionally, DSC-MRP is not universally used among clinicians and radiation oncologists, and its use is at the discretion of the managing neuro-oncology team, decided upon by a case-by-case basis. The exclusion of those without perfusion imaging means our results are only generalizable to a limited set of patients and not to all those with treated HGG. Finally, the duration of time between presentation and treatment and between treatment and DSC-MRP varied because of deviations or interruptions from the standard treatment plans due to patient intolerance of side effects or worsening of symptoms. Likewise, the decision of when to perform DSC-MRP was physician-dependent. Since the initiation of the use of MRP at our institution in 2011, the propensity to order this test has changed, adding another layer of variability: DSCMRP has become a more frequently used test and is now more 
readily available early in treatment monitoring. Unfortunately, it is difficult to account for the change in practice over the last 4 years, and the effect of these outliers on our results remains uncertain. Our study was to look at the use of DSC-MRP perfusion in the most clinically accurate scenario, and as such, this variability in timing of the test was inevitable. It would be prudent, however, for future research to look at when DSCMRP is performed both relative to the time of patient presentation and the amount of time post treatment, not only to minimize bias, but also to help establish guidelines for when these surveillance tests should be performed in the post-treatment period.

Given these limitations, our conclusions should be drawn with care; but the results demonstrate the utility of DSC-MRP as a biomarker of recurrent HGG and validate its use and subjective interpretation in today's current clinical practice. More importantly, we were able to define two separate survival curves, with DSC-MRP serving as an independent predictor of prognosis and potentially having a role in serial tumor surveillance over conventional MRI. Our positive results are of relevance to treatment and warrant the pursuit of larger, prospective studies to validate the findings.

\section{FUNDING}

The corresponding author received funding from the Regional Medical Associates Award in the amount of \$5500 from Faculty of Health Sciences at McMaster University. This award is given to a medically qualified post-graduate trainee to support original research.

\section{CONFLict of INTEREST}

None.

\section{Statement of Authorship}

CF: Conception and research planning, patient accrual/data acquisition, analysis, and manuscript writing. SP: Stastistical analysis and manuscript writing. BY: Data acquisition, analysis, and manuscript writing. ST: Data acquisition, analysis, and manuscript writing. JG: Supervisory role, conception and research planning, patient accrual/data acquisition, analysis, and manuscript writing.

\section{REFERENCES}

1. Wen P, Kesari S. Malignant gliomas in adults. N Engl J Med. 2008;359(5):492-507. doi: 10.1056/NEJMc086380.

2. Fisher JL, Schwartzbaum JA, Wrensch M, Wiemels JL. Epidemiology of brain tumors. Neurol Clin. 2007;25(4):867-90. doi: 10.1016/ j.ncl.2007.07.002.

3. Stupp R, Mason WP, Van Den Bent MJ, et al. Radiotherapy plus concomitant and adjuvant temozolomide for glioblastoma. N Engl J Med. 2005;352(10):987-96. doi: 10.1016/j.canrad.2005. 05.001.

4. Tsao MN, Mehta MP, Whelan TJ, et al. The American Society for Therapeutic Radiology and Oncology (ASTRO) evidence-based review of the role of radiosurgery for malignant glioma. Int $\mathbf{J}$ Radiat Oncol Biol Phys. 2005;63(1):47-55. doi: 10.1016/ j.ijrobp.2005.05.024

5. Butowski NA, Sneed PK, Chang SM. Diagnosis and treatment of recurrent high-grade astrocytoma. J Clin Oncol. 2006;24(8): 1273-80. doi: 10.1200/JCO.2005.04.7522.
6. Wen PY, Macdonald DR, Reardon DA, et al. Updated response assessment criteria for high-grade gliomas: response assessment in neuro-oncology working group. J Clin Oncol. 2010; 28(11):1963-72. doi: 10.1200/JCO.2009.26.3541.

7. Shah R, Vattoth S, Jacob R, et al. Radiation necrosis in the brain: imaging features and differentiation from tumor recurrence. RadioGraphics. 2012;32(5):1343-59.

8. Mullins ME, Barest GD, Schaefer PW, Hochberg FH, Gonzalez RG, Lev MH. Radiation necrosis versus glioma recurrence: conventional MR imaging clues to diagnosis. Am J Neuroradiol. 2005;26(8):1967-72. http://www.ajnr.org/cgi/content/long/26/8/ 1967. accessed January 3, 2015.

9. Brandsma D, Stalpers L, Taal W, Sminia P, van den Bent MJ. Clinical features, mechanisms, and management of pseudoprogression in malignant gliomas. Lancet Oncol. 2008;9(5):453-61. doi: 10.1016/S1470-2045(08)70125-6.

10. Mangla R, Ginat DT, Kamalian S, et al. Correlation between progression free survival and dynamic susceptibility contrast MRI perfusion in WHO grade III glioma subtypes. J Neurooncol. 2014;116(2):325-31. doi: 10.1007/s11060-013-1298-9.

11. Geer CP, Simonds J, Anvery A, et al. Does MR perfusion imaging impact management decisions for patients with brain tumors? A prospective study. Am J Neuroradiol. 2012;33(3):556-62. doi: 10.3174/ajnr.A2811.

12. Sanz-Requena R, Revert-Ventura A, Martí-Bonmatí L, AlberichBayarri Á, García-Martí G. Quantitative MR perfusion parameters related to survival time in high-grade gliomas. Eur Radiol. 2013;23(12):3456-65. doi: 10.1007/s00330-013-2967-y.

13. Asao C, Korogi Y, Kitajima M, et al. Diffusion-weighted imaging of radiation-induced brain injury for differentiation from tumor recurrence. Am J Neuroradiol. 2005;26(6):1455-60. http:// www.ajnr.org/cgi/content/long/26/6/1455. accessed January 3, 2015.

14. Barajas RFJ, Chang JS, Segal MR, et al. Differentiation of recurrent glioblastoma multiforme from radiation necrosis after external beam radiation therapy with dynamic susceptibility-weighted contrast-enhanced perfusion MR imaging. Radiology. 2009;253(2):486-96. doi: 10.1148/radiol.2532090007/-/DC1.

15. Plotkin M, Eisenacher J, Bruhn H, et al. 123 I-IMT SPECT and $1 \mathrm{H}$ MR-spectroscopy at 3.0 $\mathrm{T}$ in the differential diagnosis of recurrent or residual gliomas: a comparative study. J Neurooncol. 2004;70(1):49-58

16. Kim YH, Oh SW, Lim YJ, et al. Differentiating radiation necrosis from tumor recurrence in high-grade gliomas: assessing the efficacy of 18F-FDG PET, 11C-methionine PET and perfusion MRI. Clin Neurol Neurosurg. 2010;112(9):758-65. doi: 10.1016/ j.clineuro.2010.06.005.

17. Verma N, Cowperthwaite MC, Burnett MG, Markey MK. Differentiating tumor recurrence from treatment necrosis: a review of neuro-oncologic imaging strategies. Neuro Oncol. 2013;15(5): $515-34$

18. Xu J-L, Li Y-L, Lian J-M, et al. Distinction between postoperative recurrent glioma and radiation injury using MR diffusion tensor imaging. Neuroradiology. 2010;52(12):1193-9. doi: 10.1007/ s00234-010-0731-4.

19. Shin KE, Ahn KJ, Choi HS, et al. DCE and DSC MR perfusion imaging in the differentiation of recurrent tumour from treatmentrelated changes in patients with glioma. Clin Radiol. 2014;69(6): e264-72. doi: 10.1016/j.crad.2014.01.016.

20. Narang J, Jain R, Arbab AS, et al. Differentiating treatment-induced necrosis from recurrent/progressive brain tumor using nonmodelbased semiquantitative indices derived from dynamic contrastenhanced T1-weighted MR perfusion. Neuro Oncol. 2011;13(9) :1037-46.

21. Seeger A, Braun C, Skardelly M, et al. Comparison of three different MR perfusion techniques and MR spectroscopy for multiparametric assessment in distinguishing recurrent high-grade gliomas from stable disease. Acad Radiol. 2013;20(12):1557-65. doi: 10.1016/j.acra.2013.09.003

22. Sugahara T, Korogi Y, Kochi M, et al. Correlation of MR imagingdetermined cerebral blood volume maps with histologic and angiographic determination of vascularity of gliomas. Am J Roentgenol. 1998;171(6):1479-86. doi: 10.2214/ajr.171.6.9843274. 
23. Xu JL, Shi DP, Dou SW, Li YL, Yan FS. Distinction between postoperative recurrent glioma and delayed radiation injury using MR perfusion weighted imaging. J Med Imaging Radiat Oncol. 2011;55(6):587-94. doi: 10.1111/j.1754-9485.2011.02315.x.

24. Danchaivijitr N, Waldman AD, Tozer DJ, et al. Low-grade gliomas: do changes in rCBV measurements at longitudinal perfusionweighted MR imaging predict malignant transformation? Radiology. 2008;247(1):170-8. doi: 10.1148/radiol.2471062089.

25. Emblem KE, Zoellner FG, Tennoe B, et al. Predictive modeling in glioma grading from MR perfusion images using support vector machines. Magn Reson Med. 2008;60(4):945-52. doi: 10.1002/ mrm.21736.

26. Hakyemez B, Erdogan C, Ercan I, Ergin N, Uysal S, Atahan S. High-grade and low-grade gliomas: differentiation by using perfusion MR imaging. Clin Radiol. 2005;60(4):493-502. doi: 10.1016/j.crad.2004.09.009.

27. Jain R, Poisson L, Narang J, et al. Genomic mapping and survival prediction in glioblastoma: molecular subclassification strengthened by hemodynamic imaging biomarkers. Radiology. 2013;267(1):212-20. doi: 10.1148/radiol.12120846.

28. Fatterpekar GM, Galheigo D, Narayana A, Johnson G, Knopp E. Treatment-related change versus tumor recurrence in high-grade gliomas: a diagnostic conundrum - use of dynamic susceptibility contrast-enhanced (DSC) perfusion MRI. Am J Roentgenol. 2012;198(1):19-26. doi: 10.2214/AJR.11.7417.

29. Hu LS, Baxter LC, Smith KA, et al. Relative cerebral blood volume values to differentiate high-grade glioma recurrence from posttreatment radiation effect: direct correlation between imageguided tissue histopathology and localized dynamic susceptibility-weighted contrast-enhanced perfusio. Am J Neuroradiol. 2009;30(3):552-8. doi: 10.3174/ajnr.A1377.

30. Gasparetto EL, Pawlak MA, Patel SH, et al. Posttreatment recurrence of malignant brain neoplasm: accuracy of relative cerebral blood volume fraction in discriminating low from high malignant histologic volume fraction. Radiology. 2009;250(3):887-96. doi: 10.1148/radiol.2502071444.

31. Lupo JM, Cha S, Chang SM, Nelson SJ. Dynamic susceptibilityweighted perfusion imaging of high-grade gliomas: characterization of spatial heterogeneity. Am J Neuroradiol. 2005;26(6): 1446-54. http://www.ajnr.org/cgi/content/long/26/6/1446. accessed January 3, 2015.

32. Kim HS, Kim J-H, Kim S-H, Cho K-G, Kim SY. Posttreatment highgrade glioma: usefulness of peak height position with semiquantitative MR perfusion histogram analysis in an entire contrastenhanced lesion for predicting volume fraction of recurrence. Radiology. 2010;256(3):906-15. doi: 10.1148/radiol.10091461.

33. Shin JH, Lee HK, Kwun BD, et al. Using relative cerebral blood flow and volume to evaluate the histopathologic grade of cerebral gliomas: preliminary results. Am J Roentgenol. 2002;179(3): 783-9. doi: 10.2214/ajr.179.3.1790783.

34. Knopp EA, Cha S, Johnson G, et al. Glial neoplasms: dynamic contrast-enhanced $\mathrm{T} 2 *$-weighted $\mathrm{MR}$ imaging. Radiology. 1999;211(3):791-8. doi: 10.1148/radiology.211.3.r99jn46791. 